\title{
From percolating to dense random stick networks: Conductivity model investigation
}

\author{
Milan Žežel]* and Igor Stanković \\ Scientific Computing Laboratory, Institute of Physics Belgrade, University of Belgrade, 11080 Belgrade, Serbia
}

\begin{abstract}
In a Monte Carlo study the conductivity of two-dimensional random stick systems is investigated from the percolation threshold up to ten times the percolation threshold density. We propose a model explicitly depending on the stick density and junction-to-stick conductance ratio. The model describes the transition from the conductivity determined by the structure of a percolating cluster to the conductivity of the dense random stick networks. The model is motivated by the observed densities of the sticks and contacts involved in the current flow. The finite-size scaling effects are also included in the description. The derived model for conductivity should be broadly applicable to the random networks of the rodlike particles.
\end{abstract}

PACS numbers: 72.80.Tm, 73.50.-h, 64.60.aq, 05.10.Ln

\section{INTRODUCTION}

Recently there has been an increasing interest in the networks of randomly distributed stick (rodlike) particles ${ }^{1-4}$, due to the development in the area of conductive nanoparticles, such as the carbon nanotubes, silicon, copper, and silver nanowires ${ }^{-5}$, and the promising applications in electronics ${ }^{6}$, optoelectronics ${ }^{7}$, and sensors ${ }^{8}$. The conventional electronic composites containing stick particles as a filler can be used as a conducting channel in the thin-film network configurations ${ }^{9-11}$. Probably, the most important characteristics of the stick networks, are the conductivity, either electric ${ }^{12}$ or therma ${ }^{13}$. The conductivity dependence on the stick density and system geometry needs to be taken into account in any device design ${ }^{14}$. The percolation models $\frac{15,16}{}$ are often used to model an onset of the high electrical conductivity in the composites consisting of the conductive sticks in the insulating matrices $1.7,17$.

The percolation theory predicts that the electrical conductivity of the composite materials with the conductive filler density $n$ above, but close to the percolation threshold $n_{c}$, increases with the density by a power scaling law $\sigma \sim\left(n-n_{c}\right)^{t}$, with the universal conductivity exponent $t \approx 1.29$ for twodimensional (2D) systems ${ }^{15}$. While the conductivity scaling law is expected to be applicable only near the percolation threshold, in many experiments the scaling law was used over a much larger range of concentration, but with the nonuniversal values of the conductivity exponent ${ }^{7,17,18}$. Hu et al. ${ }^{7}$ obtained the nonuniversal value 1.5 for the conductivity exponent using the conductivity scaling law for fitting the experimental data for ultrathin carbon nanotube networks operating from the percolation threshold up to about ten times the percolation threshold density. Several numerical studies confirmed the observed nonuniversality of the conductivity exponent when the stick density is well above the percolation threshold ${ }^{19-21}$. Keblinski et al ${ }^{19}$ demonstrated that the universal power law holds from the percolation threshold $n_{c}$, to about twice its value $2 n_{c}$. For higher stick density, $n>2 n_{c}$, and in two limiting cases they observed that the conductivity scaling exponent becomes: (i) slightly higher than 1 when the junctions are superconductive and only the stick conductance is the limiting factor for the current flow through the system and (ii) close to 1.75 when the sticks are supercon- ductive and the contact conductance is the limiting factor. $\mathrm{Li}$ et $a l .20$ showed that the conductivity exponent significantly varies with the junction-to-stick conductance ratio for lower stick densities up to $2 n_{c}$. The broad range applicability of the conductivity scaling law was explained by the presence of the long-range correlations in the distribution of the conductive sticks in the system ${ }^{22}$. We will demonstrate that the nonuniversality of the conductivity exponents is a consequence of a transition from the percolating to dense stick networks.

In this paper, we numerically investigate the conductivity of the stick systems from the percolation threshold up to ten times the percolation threshold density. We show that it is not appropriate to use a simple scaling law to describe the conductivity dependence on the density both for finite and dense systems. Based on the Monte Carlo simulation results, a model is proposed describing the conductivity dependence on the stick density and the different junction-to-stick conductance ratios. The proposed model is valid for the different stick-like nanoparticles (e.g., the carbon nanotubes and the nanowires). The model is motivated by the observed structural characteristic (i.e., the density of the total sticks and contacts involved in the current flow through the system). The finite-size effects, especially pronounced in the vicinity of the percolation threshold, are included in the generic description for the conductivity of stick systems.

\section{NUMERICAL METHOD}

Monte Carlo (MC) simulations are coupled with an efficient iterative algorithm implemented on the grid platform and used to investigate the conductivity of stick systems $\mathrm{s}^{20,23,24}$. We have considered the two-dimensional systems with isotropically placed widthless sticks of length $l$. The centers of the sticks are randomly positioned and oriented inside the square system with size $L$. Two electrodes (i.e., conducting bars) are placed at the left and right sides. The top and bottom boundaries of the system are free and nonconducting. The free boundary conditions are more consistent with the finitesize rodlike nanoparticle networks in practice ${ }^{7-11}$. Two sticks lie in the same cluster if they intersect. The system percolates (conduct) if the electrodes are connected with the same cluster. The behavior of the stick percolation is studied in 
terms of the stick density $n=N /(L / l)^{2}$, where $N$ is the total number of sticks and $L / l$ is the normalized system size. The percolation threshold of the infinite system is defined by the critical density $n_{c} \approx 5.63726$ (Refs, $\underset{25,26}{ }$ ). To evaluate the conductivity of the stick systems we introduce two different conductances: (1) the conductance of the entire stick $G_{s}$ and (2) the conductance due to the stick-to-stick junction $G_{j}$. We assume diffusive electrical transport through the stick typical for the rodlike nanostructures (carbon nanotubes and nanowires) whose length is larger than the mean free path of the electrons ${ }^{27}$. According to the diffusive electrical transport the electrical resistance of a stick segment is proportional to the length of the segment ${ }^{28}$. In our simulations, each stickstick junction is modeled by an effective contact conductance regardless of the type of the junction, following the simplified approach of the authors of Refs $\frac{6,14,20}{}$. Therefore, if two sticks intersect a junction with the fixed conductance $G_{j}$ is created at the intersection point. If a stick intersects an electrode the potential of the electrode is applied to the intersection point. Kirchhoff's current law was used to balance the current flow through each node of the created network. An iterative equation solver (i.e., conjugate gradient method with Jacobi preconditioner) has been employed to solve a large system of the linear equations following from the current law ${ }^{20,29}$. After obtaining the total current $I$ under an applied voltage $V$ the macroscopic electrical conductivity of the system is evaluated as $\sigma=I / V\left(\operatorname{Ref} \frac{30}{\underline{b}}\right)$. Monte Carlo simulations have been performed for a wide range (i.e., $G_{j} / G_{s}=0.001$ to 1000) of junction-to-stick conductance ratios (cf. Refs ${ }^{31-36}$ ). Finally, for each set of the system parameters, the electrical conductivity is averaged over the $N_{\mathrm{MC}}$ independent MC realizations. To obtain the same precision for the finite-size systems $N_{\mathrm{MC}}=64000$ realizations are used for the systems with normalized size $L / l=10$ down to $N_{\mathrm{MC}}=4000$ for the largest system $L / l=40$ studied. Using the appropriate functions for the fitting data and the least-squares fitting methodology $\frac{26}{6}$, good fits with high correlation factors $\left(R^{2}>0.998\right)$ were obtained for all analyzed systems.

\section{RESULTS AND DISCUSSION}

As already mentioned, the numerical estimates of the conductivity exponent $t$ are based on the linear fit of the MC results for the logarithms of the conductivity $\sigma$ and density $n-n_{c}\left(\operatorname{Refs}^{1,19-21}\right)$. The estimates therefore rely on the assumption that $\sigma$ obeys the simple power-law dependence over a quite extended density range. As there exists no justification of such an assumption, we have investigated in detail the sbehavior of the conductivity $\sigma$ as we move away from the critical point. A local (density dependent) conductivity exponent is defined as $t(n)$ by ${ }^{37.38}$

$$
t(n)=\frac{n-n_{c}}{\sigma} \frac{d \sigma}{d n}
$$

The dependence of the local conductivity exponent $t(n)$ on the stick density $n$ and the ratio of the stick-stick junction conductance $\left(G_{j}\right)$ to stick conductance $\left(G_{s}\right)$ (i.e., $\left.G_{j} / G_{s}\right)$ is shown in Fig. 1. As one can see from a coarse observation, when the stick density approaches the percolation threshold $n_{c}$ from above the local conductivity exponent converges to the universal value for $2 \mathrm{D}$ systems $t\left(n_{c}\right) \approx 1.29$ for all $G_{j} / G_{s}$ values. The fine behavior of the local conductivity exponent for finitesize systems in the vicinity of the percolation threshold will be discussed later in this section. With the increasing concentration $n$, the local conductivity exponents $t(n)$ change quickly from the universal value $t\left(n_{c}\right)$, taking the values in a wide range $1 \leq t(n) \leq 2$. From Fig. 1, one can see that the local conductivity exponents $t(n)$ for the conductance ratio higher than $2\left(G_{j} / G_{s}>2\right)$ is a monotonically decreasing function of the stick density $n$ which converges to 1 from above. Somewhat surprisingly, for the conductance ratios lower than 1 (i.e., $G_{j} / G_{s}<1$ ), the local exponent $t(n)$ is not a monotonic function and has a local maximum. The observed density where the local conductivity exponent reaches a maximum is decreasing with the conductance ratio $G_{i} / G_{c}$.

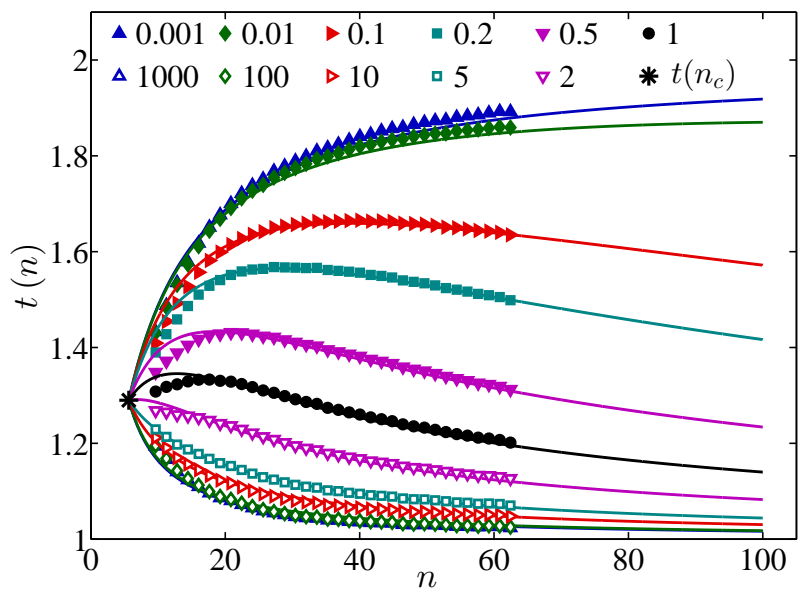

FIG. 1. (Color online) The dependence of the local conductivity exponent $t(n)$ on the stick density $n$ and junction-to-stick conductance ratio $G_{j} / G_{s}$. The points are MC simulation results obtained using Eq. 11 for the system size $L / l=20$. The values are given for the conductance ratios $G_{j} / G_{s}=0.001,0.01,0.1,0.2,0.5,1$ (filled), and their inverse values $1000,100,10,5,2$ (transparent). The error bars are smaller than the size of the points. The star marker denotes the expected universal value for the conductivity exponent at the percolation threshold $t\left(n_{c}\right)$. The lines represent the local conductivity exponents $t(n)$ obtained from the conductivity model for an infinitesize system, Eq. (3).

To explain the observed behavior of the exponent $t(n)$ at the higher densities $n>2 n_{c}$, one needs to look into the structure of the dense conducting stick systems. Figure 2 shows the densities of the sticks $n^{I}$ and junctions $n_{j}^{I}$ that carry the current through the system. For sufficiently high stick densities $\left(n>2 n_{c}\right)$, one can see that almost all the sticks and junctions in the system contribute to the conductivity and that the density of the current-carrying junctions increases with the stick density $n$ by a square power law $n_{j}^{I} \sim n^{2}$. The reason for this is that the mean number of contacts per stick is proportional to the stick density, see Ref $\frac{39}{9}$. Also, for a sufficiently high stick density $n$ the current-carrying stick density $n^{I}$ is proportional to $n$. Therefore, when the stick density is well above 
the percolation threshold $n_{c}$, the conductivity of the system can be modeled as an equivalent serial conductance $n$ sticks in parallel and $n^{2}$ junctions in parallel

$$
\sigma \sim \frac{1}{b n^{-1} / G_{s}+n^{-2} / G_{j}}
$$

where $b$ is a constant parameter. One can see that the square term $n^{-2} / G_{j}$, originating from the junctions, converges faster to zero than the linear term $b n^{-1} / G_{s}$. This explains the conductivity exponent $t(n)$ approaching to 1 when the stick density is sufficiently high (i.e., $n \gg G_{s} / G_{j}$ ) and the existence of the local exponent maximum in Fig. 1. If the sticks are much more conductive than the junctions (e.g., $G_{j} / G_{s}=0.01$ ) the density where the local conductivity exponent starts to converge to 1 is high and computationally unreachable in the $\mathrm{MC}$ simulations shown in Fig. 1. Only in the limiting case when the sticks are superconductive and the conductance ratio approaches zero, i.e., $G_{j} / G_{s} \rightarrow 0$, should the conductivity exponent $t(n)$ converge to 2 with the increasing density $n$, which is consistent with Keblinski et al. ${ }^{19}$. In the other limit, when the junctions are superconductive (i.e., $G_{j} / G_{s} \rightarrow \infty$ ) the local conductivity exponent $t(n)$ should have the fastest convergence to 1 .

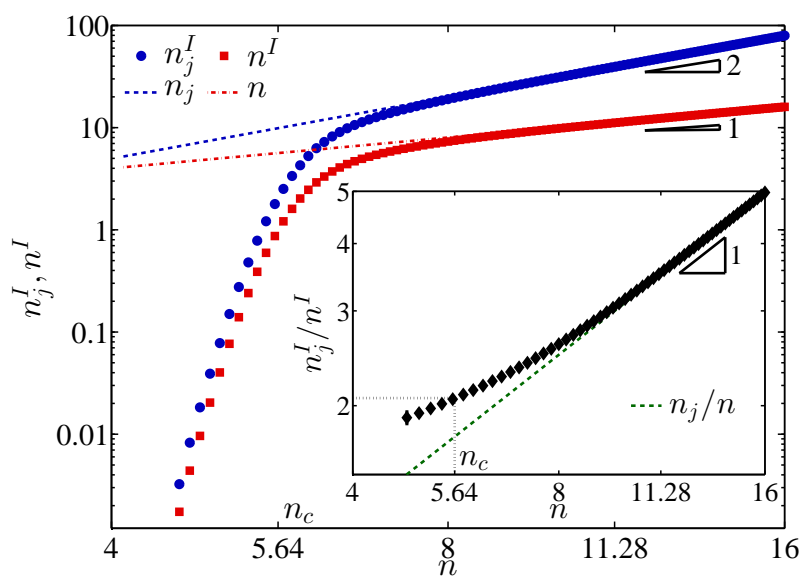

FIG. 2. (Color online) The density of junctions $n_{j}^{I}$ and sticks $n^{I}$ involved in the current flow through the system is compared with the density of all junctions $n_{j}$ and sticks $n$ in the system of size $L / l=20$. For higher stick densities $n$ almost all junctions and sticks will carry some current. The error bars are smaller than the size of the points. Inset: The density ratio of the current-carrying junctions to current-carrying sticks $n_{j}^{I} / n^{I}$ is higher than the density ratio of all junctions to all sticks $n_{j} / n$ in the system. At the percolation threshold this ratio is about 2 [i.e., $n_{j}^{I} / n^{I}=2.0(1)$ ].

At the densities close to the percolation threshold $n_{c}$, only a fraction but not all the sticks and junctions in the system contribute to the conductivity, by carrying some current. From Fig. 2 (inset), one can see that at the percolation threshold $n_{c}$, the density of the current-carrying junctions is about two times higher than the density of the current-carrying sticks [i.e, $\left.n_{j}^{I} / n^{I}=2.0(1)\right]$. From the framework of the percolation theory we cannot determine a density-dependent factor of proportionality in the conductivity power law [i.e., $\left.\sigma \sim\left(n-n_{c}\right)^{t}\right]$. Instead, we fit the factor of proportionality with an expression for the dense systems [i.e., Eq. (2)] and obtain $1 /\left[b n^{t-1} / G_{s}+\left(n+n_{c}\right)^{t-2} / G_{j}\right]$. This relation explicitly includes the previous observation that there is almost exactly two times more current-carrying junctions than current-carrying sticks at the percolation threshold. For a general conductivity description of the infinite-size systems we obtain

$$
\sigma=a \frac{\left(n-n_{c}\right)^{t}}{b n^{t-1} / G_{s}+\left(n+n_{c}\right)^{t-2} / G_{j}},
$$

where $a=0.027(1)$ and $b=0.061(3)$ are fitting parameters calculated using the least-squares fitting methods. The solid lines in Fig. 1 denote the local conductivity exponents $t(n)$ calculated from Eq. (1), using the model for an infinite system given by Eq. (3), for a wide range of conductance ratios $G_{j} / G_{s}=0.001$ to 1000 . Deviations between the modeled and MC values for local conductivity exponent $t(n)$ are comparable to the statistical errors.

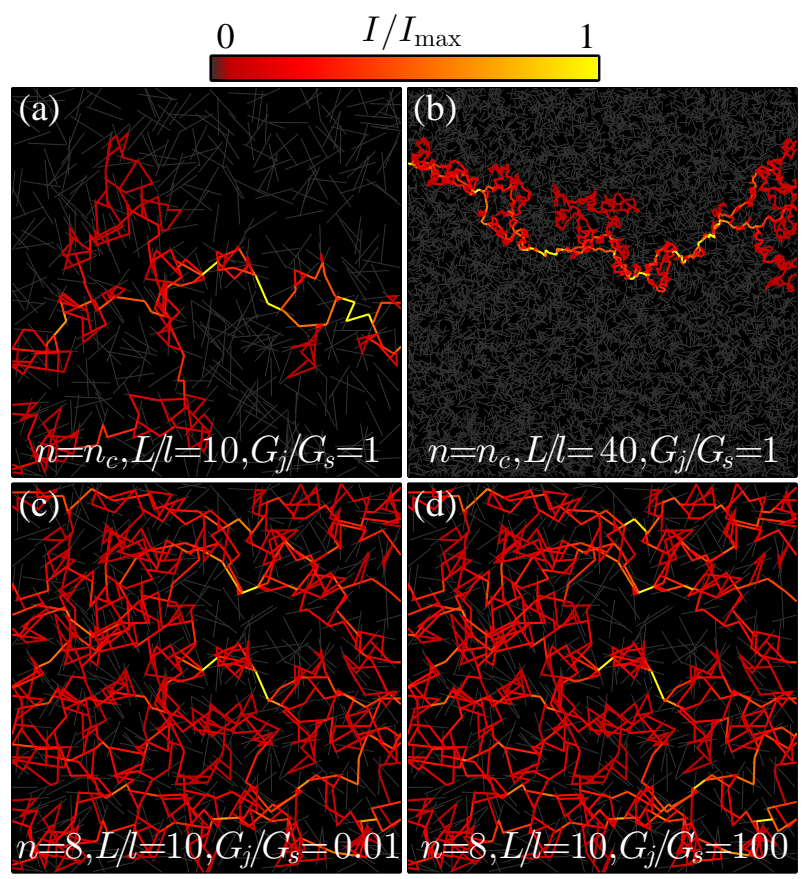

FIG. 3. (Color online) Simulated current (a) and (b) at different system sizes and (c) and (d) junction-to-stick conductance ratios. The current through a stick $I$ is given relative to the maximal current in the system $I_{\max }$. There is a large difference in the fraction of the system involved in the current flow between the two nominally identical films in term of density ( $n=n_{c}$ ) and junction-to-stick conductance ratio $\left(G_{j} / G_{s}=1\right)$ for two different system sizes $L / l=10$ and 40. The current redistribution with the increasing junction-to-stick conductance ratio $G_{j} / G_{s}$ is visible from (c) and (d). (c) If junctions are weakly conductive (i.e., $G_{j} / G_{s}=0.01$ ) the maximal current is flows along the shortest path with the least junctions. (d) For high junction conductance values (i.e. $G_{j} / G_{s}=100$ ), the total current is evenly carried by the larger number of shortest paths connecting electrodes. This effect is only visible at higher densities (e.g., $n=8$ ) where several paths connecting electrodes exist. 


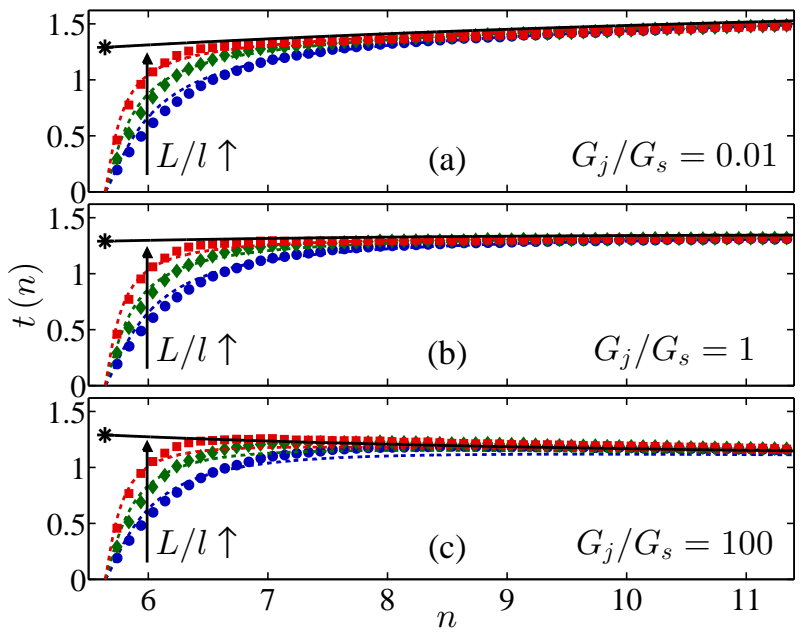

FIG. 4. (Color online) The local conductivity exponents $t(n)$ for the stick systems with increasing size $L / l=10,20$, and 40 and for three conductance ratio values (a) $G_{j} / G_{s}=0.01$, (b) 1 , and (c) 100 . The direction of the increase of $L / l$ is indicated on the graphs. The points are obtained from the MC simulations and calculated using Eq. (1). The error bars are smaller than the size of the points. The solid line represents the local conductivity exponent $t(n)$ for the infinite system obtained from Eq. (3), while the dashed lines denote the local conductivity exponents $t(n)$ obtained from the model that includes finite-size effects, Eq. (7). The star marker denotes the expected value for the conductivity exponent of the infinite system at the percolation threshold $n_{c}$.

Figure 3 illustrates the structure of the percolating cluster [Figs. 3 (a) and 3 (b)] and the redistribution of the current in the dense stick networks due to the junction-to-stick conductance ratio increase [Figs. 3 (c) and 3 (d)]. The current through a stick $I$ is given relative to the maximal current in the system $I_{\max }$. As one can see from Figs. 3 (a) and 3 (b), the percolating cluster consists of a few sub-clusters connected by high current links. This explains why on average more junctions than sticks are needed to shortcut the electrodes. For a large, but finite-size systems at the percolation threshold, the density of the current-carrying junctions decreases as $n_{j}^{I} \sim(L / l)^{-\beta / \nu}$ with normalized system size $L / l$, where $\beta=5 / 36$ for $2 \mathrm{D}$ systems 15 . Also, the density of the current-carrying sticks at the percolation threshold is $n^{I} \sim(L / l)^{-\beta / \nu}$. As a result the densities of the current-carrying sticks and junctions decrease with system size [cf. Figs. 3 a) $(L / l=10)$ and 3 b) $(L / l=40)]$. Furthermore, the density ratio $n_{j}^{I} / n^{I}$ at the percolation threshold converges to a constant value with the increase of the system size, see Fig. 2. At higher stick densities (i.e., $n=8$ ) one can see that current flows along many parallel paths connecting electrodes. An increase of the junction-tostick conductance ratio $G_{j} / G_{s}$ results in the more uniform redistribution of the current [cf. Figs. 3(c) $\left(G_{j} / G_{s}=0.01\right)$ and 3(d) $\left.\left(G_{j} / G_{s}=100\right)\right]$. For weakly conductive junctions (i.e., low conductance ratio $G_{j} / G_{s}=0.01$ ), most of the current flows through a shortest path with the least junctions along. With the increase of the junction conductance several parallel paths become visible. As a result, the total current through the system is more evenly distributed, resulting in the higher

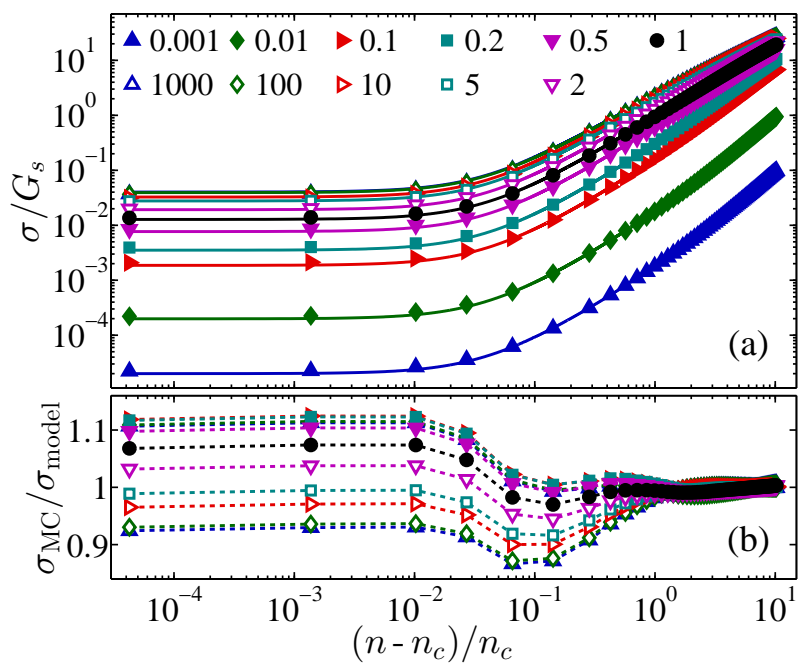

FIG. 5. (Color online) (a) Conductivity as a function of $\left(n-n_{c}\right) / n_{c}$ is obtained from the MC simulations (points) for the stick system of size $L / l=20$ and the junction-to-stick conductance ratio from $G_{j} / G_{s}=0.001$ to 1000 (from bottom to top). The lines denote values obtained from the conductivity model for the finite-size systems given by Eq. (7). (b) The conductivity ratio between the MC simulation results $\sigma_{\mathrm{MC}}$ and the values obtained from the model $\sigma_{\text {model }}$ for corresponding finite-size systems, Eq. (7). The error bars are smaller than the size of the points.

conductivity. This is also expected from Eq. (3).

If we compare the infinite system model prediction and MC simulation results in Fig. 1 close to the percolation threshold, we observe a deviation between the predicted and simulated values. This deviation is a result of the finite-size effects, since the MC results in Fig. 1 are calculated for the large but finitesize system (i.e, $L / l=20$ ). The convergence of the local conductivity exponents with the increasing system size is shown in Fig. 4. The points are MC simulation results for the systems with sizes $L / l=10,20$, and 40 and the solid line denotes the model for an infinite system given by Eq. (3). For the finite-size systems close to the percolation threshold we observe a large deviation of the local conductivity exponent $t(n)$ from the model. The local conductivity exponent decreases with the decreasing system size and can be even lower then $1[t(n)<1]$. This is result of a nonzero conductivity value for the finite-size systems at the percolation threshold 15. Therefore, the model should be adapted for the finite-size systems. The finite-size scaling arguments $15,26,40$ suggest that the conductivity $\sigma$ depends on the system size $L$ as

$$
\sigma \sim\left(n-n_{c}\right)^{t} f\left[\frac{\xi(n)}{L}\right]
$$

where $\xi(n) \sim l\left|n-n_{c}\right|^{-\nu}$ is the correlation length that measures the linear extent of the largest cluster. For 2D systems the correlation-length exponent is $\nu=4 / 3\left(\operatorname{Ref}^{15}\right)$. For the infinite system above the percolation threshold [i.e., $\xi(n) / L \rightarrow 0$ ] the conductivity follows the simple scaling law and finite-size scaling function $f[\xi(n) / L]$ converges to a constant value. In the other limit, for the finite-size systems at 
the percolation threshold [i.e. $\xi(n) / L \rightarrow \infty$ ], conductivity has a nonzero value $\sigma \sim(L / l)^{-t / \nu}\left(\operatorname{Ref}{ }^{15}\right)$. Therefore, the finite-size scaling function should have a form $f[\xi(n) / L] \sim$ $[\xi(n) / L]^{t / \nu}$, to cancel the conductivity dependence on density in Eq. (4). Since the finite-size scaling function $f[\xi(n) / L]$ above the percolation threshold is a continuous and smooth function $\stackrel{15}{ }$, we approximate it by a combination of its two limiting behaviors

$$
f\left[\frac{\xi(n)}{L}\right] \sim 1+c\left(n-n_{c}\right)^{-t}(L / l)^{-t / \nu},
$$

where $c$ is the finite-size parameter. Inserting Eq. (5) into Eq. (4) the first-order approximation of the finite-size scaling law for conductivity becomes

$$
\sigma \sim\left(n-n_{c}\right)^{t}+c(L / l)^{-t / \nu} .
$$

Finally, incorporating the finite-size effects given by Eq. (6) into the conductivity model for an infinite-size system, Eq. (3), we obtain the finite-size model for conductivity

$$
\sigma=a \frac{\left(n-n_{c}\right)^{t}+c(L / l)^{-t / \nu}}{b n^{t-1} / G_{s}+\left(n+n_{c}\right)^{t-2} / G_{j}} .
$$
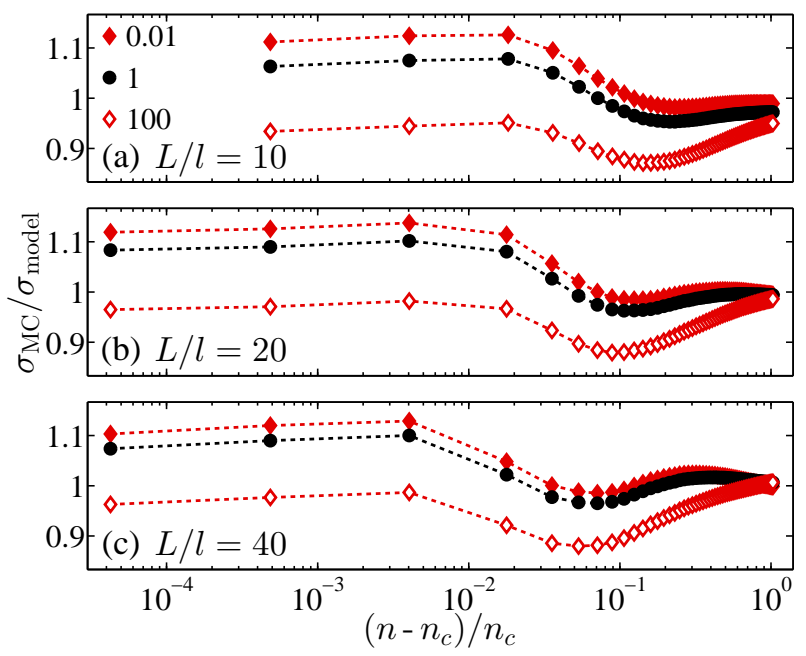

FIG. 6. (Color online) The conductivity ratio between the MC simulation results $\sigma_{\mathrm{MC}}$ and the values obtained from the model $\sigma_{\text {model }}$ given by Eq. (7) for different system sizes (a) $L / l=10$, (b) 20 , and (c) 40 and for three conductance ratio values $G_{j} / G_{s}=0.01,1$, and 100. The error bars are smaller than the size of the points.

The finite-size parameter for 2D stick systems $c=2.5(1)$ is calculated using the least squares fitting methods. A comparison between the $\mathrm{MC}$ results and the values obtained from the model given by Eq. (7) is shown in Fig. (4) The dashed lines in Fig. 4 denote the local conductivity exponents $t(n)$ calculated from the model including finite-size effects, Eq. (7), for systems with increasing size $L / l=10,20$, and 40 and for three conductance ratio values $G_{j} / G_{s}=0.01$, 1 , and 100 . We see that the introduction of finite-size effects in the model significantly improves the quantitative description of the system close to the percolation threshold. Finally, the MC conductivity values normalized with the stick conductance $G_{s}$ and fitted by Eq. (7) for the systems of size $L / l=20$ and conductance ratios from $G_{j} / G_{s}=0.001$ to 1000 are shown in Fig. [5 a). For all studied values of the conductance ratio $G_{j} / G_{s}$ the conductivity obtained from the model agrees with the $\mathrm{MC}$ results over the whole range of the stick density $n$, see Fig. 5 a). The agreement between the MC results and the model is good for higher stick densities $\left(n>2 n_{c}\right)$ (i.e., further away from the percolation threshold), but not so good in the vicinity of the percolation threshold [cf. Fig. 5(b)]. Hence, in the vicinity of the percolation threshold the conductivity ratio between the MC simulation results and the values obtained from the model is shown in Fig. 6for different system sizes $L / l=10,20$, and 40. For all three system sizes in Fig. 6 the curves look qualitatively similar. Only the density where the dense-system behavior becomes dominant decreases with the system size $L / l$. To improve the agreement between the MC results and the model close to the percolation threshold one could consider a further refinement of the model to include higher-order correction for the finite-size effect. Finally, the proposed model for conductivity gives a good estimate of the local conductivity exponents, as one can see in Figs. 1 1 and 4.

\section{CONCLUSIONS}

In this paper, we present the results of the numerical Monte Carlo study of the conductivity of random stick systems for the wide range of densities and junction-to-stick conductance ratios. We observe the transition from the conductivity of the percolating cluster to the conductivity of the dense random stick networks with increasing density. Three limiting cases are identified for the conductivity of whole system: one in the vicinity of the percolation threshold, and two for high densities when either the junctions or sticks are superconductive. Each of these cases has a different exponent governing the power-law dependence of the conductivity from density (i.e., 1.29, 1, and 2, respectively). As result, the exponent can take values anywhere in the range $(1,2)$ depending on the junction-to-stick conductance ratio. For finite-size systems the density-dependent exponent can even take values lower than 1. Therefore, it is not appropriate to use a simple scaling law to describe the conductivity dependence on the density both for finite and dense systems. We instead propose a comprehensive conductivity model, derived from the behavior of the limiting cases. We find that the proposed description gives a satisfactory estimation of the conductivity and the local conductivity exponent (which is related to the first derivative of the conductivity) over the whole range of the stick density values. Finite-size effects, important for many practical realizations of the random conducting networks, are also included in the conductivity model. The presented methodology could be used to describe the properties of other conducting systems (i.e., disks, spheres, and fibers).

The authors acknowledge support by the Ministry of Science of the Republic of Serbia, under Projects No. ON171017 and No. III45018. Numerical simulations were run on the 
AEGIS e-Infrastructure, supported in part by FP7 projects EGI-InSPIRE, PRACE-1IP, PRACE-2IP, and HP-SEE. The authors also acknowledge support received through SCOPES Grant No. IZ73Z0-128169 of the Swiss National Science Foundation.
* Corresponding author:milan.zezelj@ipb.ac.rs

${ }^{1}$ R. Ramasubramaniam, J. Chen, and H. Liu, Appl. Phys. Lett. 83, 2928 (2003).

2 X.-Z. Bo, N. G. Tassi, C. Y. Lee, M. S. Strano, C. Nuckolls, G. B. Blanchet, Appl. Phys. Lett. 87, 203510 (2005).

3 S. Seppälä, E. Häkkinen, M. J. Alava, V. Ermolov, and E. T. Seppälä, Europhys. Lett. 91, 47002 (2010).

${ }^{4}$ V. K. Sangwan, A. Behnam, V. W. Ballarotto, M. S. Fuhrer, A. Ural, and E. D. Wiliams, Appl. Phys. Lett. 97, 043111 (2010).

5 S. M. Bergin, Y.-H. Chen, A. R. Rathmell, P. Charbonneau, Z.-Y. Lib, and B. J. Wiley, Nanoscale 4, 1996 (2012).

${ }^{6}$ S. Kumar, J.Y. Murthy, and M. A. Alam, Phys. Rev. Lett. 95, 066802 (2005).

${ }^{7}$ L. Hu, D. S. Hecht, and G. Grüner, Nano Lett. 4, 2513 (2004).

${ }^{8}$ Q. Cao, H.-S. Kim, N. Pimparkar, J. P. K., C. W., M. Shim, K. Roy, M. A. Alam, and J. A. Rogers, Nature (London) 454, 495 (2008).

9 S. Kumar, G. B. Blanchet, M. S. Hybersten, J. Y. Murthy, and M. A. Alam, Appl. Phys. Lett. 89, 143501 (2006).

${ }^{10}$ M. Engel, J. P. Small, M. Steiner, M. Freitag, A. A. Green, M. C. Hersam, and P. Avouris, ACS Nano 2, 2445 (2008).

11 B. Chandra, H. Park, A. Maarouf, G. J. Martyna, and G. S. Tulevski, Appl. Phys. Lett. 99, 072110 (2011).

12 Tao Hu, A. Yu. Grosberg, and B. I. Shklovskii, Phys. Rev. B 73, 155434 (2006).

13 A. N. Volkov and L. V. Zhigilei, Phys. Rev. Lett. 104, 215902 (2010).

14 A. Behnam and A. Ural, Phys. Rev. B 75, 125432 (2007).

15 D. Stauffer and A. Aharony, Introduction to Percolation Theory, 2nd revised ed. (Taylor and Francis, London, 2003).

16 I. Balberg, N. Binenbaum, and C. H. Anderson, Phys. Rev. Lett. 51, 1605 (1983).

${ }^{17}$ H. E. Unalan, G. Fanchini, A. Kanwal, A. D. Pasquier, and M. Chhowalla, Nano Letters 6, 677 (2006).

18 T. W. Noh, S.-I. Lee, Y. Song, and J. R. Gaines, Physics Lett. 114A, 207 (1985).

19 P. Keblinski and F. Cleri, Phys. Rev. B 69, 184201 (2004).

20 J. Li and S.-L. Zhang, Phys. Rev. E . 81, 021120 (2010).

21 Y. Hazama, N. Ainoya, J. Nakamura, and A. Natori, Phys. Rev. B 82, 045204 (2010).

22 M. Sahimi, Heterogeneous Materials I: Linear Transport and Optical Properties (Springer-Verlag, New York, 2003).

${ }^{23}$ I. Stanković, M. Kröger and S. Hess, Comp. Physics Comm. 145, 371 (2002)

${ }^{24}$ A. Balaž, I. Vidanović, D. Stojiljković, D. Vudragović, A. Belić, and A. Bogojević, Commun. Comput. Phys. 11, 739 (2012).

25 J. Li and S.-L. Zhang, Phys. Rev. E . 80, 040104 (2009).

${ }^{26}$ M. Žeželj, I. Stanković, and A. Belić, Phys. Rev. E 85, 021101 (2012).

27 Y. Zhou, S. Sreekala, P. M. Ajayan, and S. K. Nayak, J. Phys.: Condens. Matter 20, 095209 (2008).

28 J.-Y. Park, S. Rosenblatt, Y. Yaish, V. Sazonova, H. Üstünel, S. Braig, T.A. Arias, P. W. Brouwer, and P. L. McEuen, Nano Lett.
4, 517 (2004).

29 J. R. Shewchuk, An Introduction to the Conjugate Gradient Method Without the Agonizing Pain (1994), http://www.cs.cmu.edu/quake-papers/painless-conjugate

30 For a rectangular system of size $L_{x} \times L_{y}$, where $L_{x}$ is the length of the electrodes and $L_{y}$ is the distance between them, the relation between the system conductance $G$ and conductivity $\sigma$ is according to Ohm's law given by $G=\sigma L_{x} / L_{y}$. In this paper the system is the square-shaped $L_{x}=L_{y}=L$, which implies $G=\sigma$. Although in the square system conductance is equal to the system conductivity, for clarity we have assumed terminology in which the conductance is used for denoting a single conductive element (stick or junction) while the conductivity is related to the entire system.

${ }^{31}$ M. S. Fuhrer, J. Nygård, L. Shih, M. Forero, Y. G. Yoon, M. S. C. Mazzoni, H. J. Choi, J. Ihm, S. G. Louie, A. Zettl, and P. L. McEuen, Science 288, 494 (2000).

32 A. Buldum and J. P. Lu, Phys. Rev. B 63, 161403 (2001).

33 J.-Y. Yu, S.-W. Chung, and J. R. Heath, J. Phys. Chem. B 104, 11864 (2000).

34 A. D. Franklin, Z. Chen, Nat. Nanotechnol. 5, 858 (2010).

35 A. Bid, A. Bora, and A. K. Raychaudhuri, Phys. Rev. B 74, 035426 (2006).

36 The choice of an extended range of conductance ratios is based on the experimental measurements on crossed rodlike nanoparticles, such as carbon nanotubes (CNT) (Refs. ${ }^{31,32}$ ) and nanowires (NW) $\left(\operatorname{Ref}^{33}\right)$. The CNTs and NWs exist as metallic or semiconducting, based on their chirality and composition (Refs. ${ }^{31,33}$ ). For crossed single-walled CNTs the junction conductance is of the order of magnitude $G_{j}^{\mathrm{MM}, \mathrm{SS}} \approx 0.1 e^{2} / h$ (where $e$ is the electron charge, $h$ is Planck's constant, and $e^{2} / h \approx 39 \mathrm{~S}$ ) for the metallic/metallic or semiconducting/semiconducting junctions and two orders lower for metallic/semiconducting junctions [i.e., $G_{j}^{\mathrm{MS}} \approx$ $\left.10^{-3} e^{2} / h\left(\operatorname{Refs}^{31}\right)\right]$. In the diffusive case, typical for CNTs and NWs whose length $l$ is larger than the mean free path of electrons $\lambda$, the conductance can be approximated by $G_{s} \approx\left(4 e^{2} / h\right)(\lambda / l)$ (Refs. ${ }^{27,28,34}$ ). For single-walled CNTs the mean free path of the electrons is of the order $\lambda \approx 1 \mu \mathrm{m}$ (Refs. ${ }^{28,34}$ ). For NWs, the mean free path is considerably shorter $\approx 40 \mathrm{~nm}\left(\operatorname{Ref}{ }^{35}\right)$, implying that the diffusive conduction model is applicable even for very short NWs. Therefore, the junction-to-stick conductance ratio $G_{j} / G_{s}$ depends on the total length of the individual stick. When the length of the stick is of the order of the mean free path of the electrons the conductance ratio is $G_{j} / G_{s}=0.001-0.1$. On the other hand, for very long CNTs and NWs (i.e., $l>100 \lambda$ ), the conductance ratio becomes higher than $1, G_{j} / G_{s}>1$.

37 J. Bernasconi, Phys. Rev. B 18, 2185 (1978).

38 C. Grimaldi and I. Balberg, Phys. Rev. Lett. 96, 066602 (2006).

39 J. Heitz, Y. Leroy, L. Hébrard, and C. Lallement, Nanotechnology 22, 345703 (2011).

40 D. B. Gingold and C. J. Lobb, Phys. Rev. B 42, 8220 (1990). 